\title{
Ultrahigh-brightness, femtosecond ArF excimer laser system
}

\author{
K. Mossavi, Th. Hofmann, and F. K. Tittel \\ Department of Electrical and Computer Engineering, Rice Quantum Institute, Rice University, \\ P.O. Box 1892, Houston, Texas 77251 \\ G. Szabo \\ Department of Optics \& Quantum Electronics, JATE University, H-6720 Szeged, Dom ter 9, Hungary
}

(Received 28 October 1992; accepted for publication 14 January 1993)

\begin{abstract}
An ultrahigh-brightness ArF excimer laser system is described that is capable of generating pulse energies of $60 \mathrm{~mJ}$ with a pulse duration of $\sim 700 \mathrm{fs}$. The system utilizes a newly developed seed pulse generation scheme based on spectrally compensated sum-frequency mixing in beta-barium metaborate (BBO), and a double-pass discharge pumped ArF excimer preamplifier followed by an electron beam pumped power amplifier.
\end{abstract}

Recent advances in ultrahigh-brightness laser technology have led to the development of laser systems with focal spot intensities in excess of $10^{19} \mathrm{~W} / \mathrm{cm}^{2}$. These extremely high intensities permit, besides a variety of very interesting applications such as generation of dense, nonequilibrium plasmas, or studying multiphoton phenomena, investigations of light-matter interaction in a new regime where the optical field strength is much larger than the Bohr electric field (a comprehensive review of the state-of-art of high intensity optics can be found in Ref. 1). In the blue and UV range of the spectrum ultrashort pulse rare gas halide excimers offer unique merits for high brightness laser systems. Femtosecond excimer laser systems with powers of up to the terawatt range have been developed based on $\mathrm{XeCl}^{2-4} \mathrm{KrF}^{5-8}$ and recently $\mathrm{XeF}(C-A)^{9}$ excimers. The ArF laser, which is a very efficient excimer laser in the nanosecond regime, can provide a means for new applications because of its short wavelength $(193 \mathrm{~nm})$. The difficulties associated with the seed pulse generation at $193 \mathrm{~nm}$ where no frequency doubling is possible with presently commercially available nonlinear crystals, and the losses of the optical materials increase with decreasing wavelength, have limited the peak output power of the ArF systems described in the literature to the $2-10 \mathrm{GW}$ range. ${ }^{10-14}$

In this work we present an ArF excimer laser system based on a newly developed, spectrally compensated sumfrequency mixing seed pulse generation scheme ${ }^{15}$ and an electron beam pumped excimer power amplifier, capable of generating nearly $100 \mathrm{GW}$ peak power with $\sim 700$ fs pulse duration.

The layout of the system is shown in Fig. 1(a). Details of the femtosecond seed pulse generation scheme are depicted schematically in Fig. 1(b). The dye laser part of the system is similar to the one developed for blue-green operation and described in detail in Ref. 16. A hybridly modelocked cw dye laser oscillator (with Pyridine 2 as laser dye and DDI as saturable absorber) pumped by $3 \mathrm{~W}$ of the second harmonic of a cw mode-locked Nd:YAG laser (Coherent Antares 76-s) produces femtosecond pulses, tunable around $707 \mathrm{~nm}$. The pulse duration of the mode-locked dye laser pulses was measured by a background-free, multiple shot autocorrelator (Inrad 5-14A) and found to be 800 fs assuming a Gaussian pulse shape. The pulses are compressed by a fiber and prism compressor to $300 \mathrm{fs}$ and amplified in a two stage dye amplifier system ${ }^{16}$ to $0.8 \mathrm{~mJ}$ pulse energy. The dye amplifier system with Pyridine 2 as amplifying medium is pumped by $50 \mathrm{~mJ}, 532 \mathrm{~nm}, \sim 100 \mathrm{ps}$ long pulses delivered by a closed-cavity regenerative amplifier (Continuum Corporation RGA-69) followed by one external Nd:YAG amplifier and a BBO frequency doubler. The regenerative amplifier, running at repetition rates between 1 and $6 \mathrm{~Hz}$, is seeded by the output of the cw modelocked Nd:YAG laser. Since the gain time of the amplifier system is much shorter than the pulse separation in the pulse train of cw mode-locked dye laser, the amplifier system also serves as a single pulse selector. The pulse duration after the dye amplifier was measured by a single shot, phase sensitive autocorrelator ${ }^{17}$ to be $300 \mathrm{fs}$.

The output of the femtosecond dye laser system along with the $\sim 50 \mathrm{ps}$ fourth harmonic pulses of the regenerative amplifier is sent into the dispersively compensated sum frequency scheme to generate the $193 \mathrm{~nm}$ seed pulses for the ArF excimer laser. To achieve a smooth intensity profile at the mixing crystal, a spatially uniform part of the perimeter of the doughnut shaped $266 \mathrm{~nm}$ beam is selected by a diaphragm. (The details of the dispersively compensated scheme are described in Ref. 15.) By using 0.8 and 3 $\mathrm{mJ}$ pulse energies at 707 and $266 \mathrm{~nm}$, respectively, the pulse energy at $193 \mathrm{~nm}$ is $12 \mu \mathrm{J}$. It is interesting to note, that taking into account the ratio of the red and UV input pulse durations this corresponds to almost $100 \%$ efficiency, i.e., practically all the energy present in the $266 \mathrm{~nm}$ pulse in the time duration of the $707 \mathrm{~nm}$ pulse is converted into energy at the sum frequency. This is supported by the fact that by decreasing the energy of the dye laser pulses by $50 \%$ the decrease of the energy at $193 \mathrm{~nm}$ was only about $10 \%$. This also means that the efficiency of the spectrally compensated sum-frequency scheme is mainly limited by the pulse duration of the $266 \mathrm{~nm}$ pulse. Consequently, by using $266 \mathrm{~nm}$ pulses of 5 ps duration or shorter which can still be realized with mode-locked Nd:YAG lasers, with the same dye laser energy of $0.8 \mathrm{~mJ}$, the pulse energy is expected to exccd $50 \mu \mathrm{J}$ at $193 \mathrm{~nm}$.

The seed pulses at $193 \mathrm{~nm}$ are first preamplified in a double-pass scheme by using a conventional discharge pumped ArF excimer laser. A Lambda Physik type EMG 101 excimer laser filled with a 150 mbar $5 \% \mathrm{~F}_{2} / \mathrm{Ar}: 210$ mbar Ar:1.84 mbar He mixture was used. To minimize the 


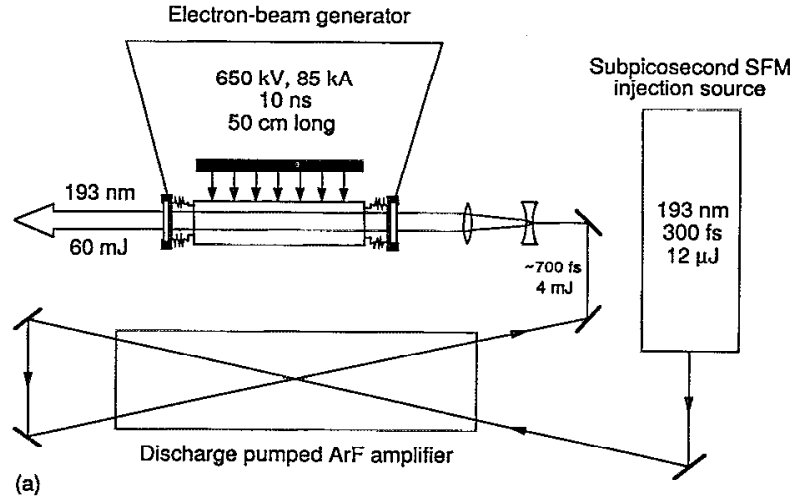

(a)

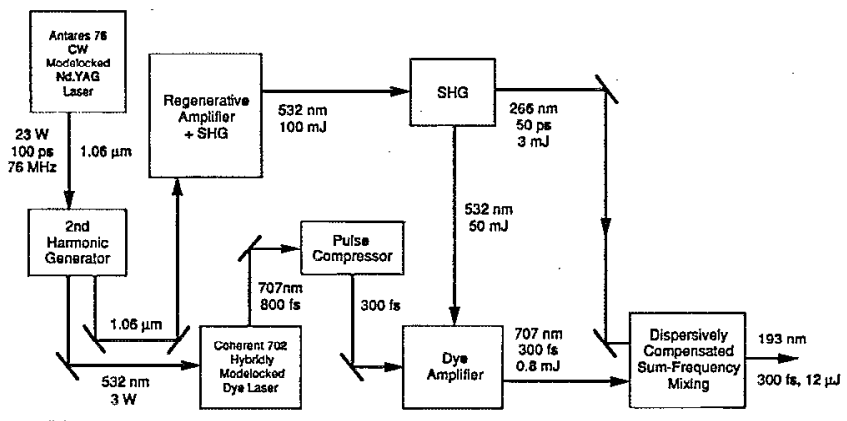

(b)

FIG. 1. The schematic layout of the ultrahigh-brightness ArF excimer laser system. (a) The discharge pumped excimer preamplifier followed by an electron beam pumped power amplifier. (b) The femtosecond seed pulse generation scheme.

amplified spontaneous emission (ASE) in the preamplifier an off-axis amplification scheme ${ }^{18}$ was employed. In our experiments it was not possible to fully match the rectangular shape of the discharge pumped amplifier to the circular aperture of the electron beam pumped final amplifier because of the unavailability of the appropriate UV cylindrical optics. Therefore, the angle between the axis of the discharge pumped amplifier tube and the beam to be amplified was chosen to be a maximum at the expense of some loss of the total output energy. (This energy decrease is a consequence of the smaller useful amplifier aperture reflecting larger off-axis angles.) With the preamplifier optimized for the maximum signal-to-noise ratio the pulse energy of the ultrashort pulses is $4 \mathrm{~mJ}$ after double-pass amplification corresponding to an energy density of $10 \mathrm{~mJ} /$ $\mathrm{cm}^{2}$, with a time integrated ASE energy of $20 \mu \mathrm{J}$. However, when the system is optimized for maximum output energy, the ultrashort pulse energy increases to $8 \mathrm{~mJ}$.

The reliability of the whole short pulse excimer laser system is strongly influenced by the timing jitter between the $193 \mathrm{~nm}$ seed pulses and the two excimer amplifiers. Optical triggering based on the seed pulses that would provide the minimal jitter cannot be used since both the discharge and electron beam pumped excimer amplifier have an inherent internal delay between 500 and $1000 \mathrm{~ns}$. This problem can, however, be solved by using an early pulse of the pulse train from the cw mode-locked Nd:YAG laser as a pretrigger. Furthermore, the pulse train from the modelocked Nd:YAG laser is synchronized to the rf driver sig-

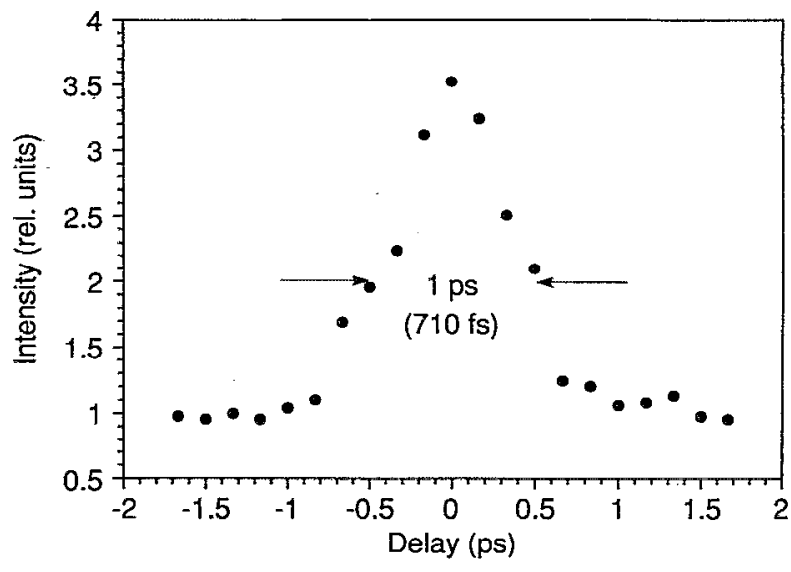

FIG. 2. The autocorrelation trace of the femtosecond ArF laser pulses.

nal of the mode locker with a jitter in the picosecond range. This synchronism is also maintained in the femtosecond dye laser oscillator because of its synchronous pump design. This means that the rf signal of the mode locker can be used as a master trigger for the whole system. A digital delay generator (Stanford Research Systems, model DG535) was triggered by the mode-locker signal. This delay generator triggered the excimer lasers, and the Pockels cell of the regenerative amplifier. In this way the jitter between the seed pulses and the discharge pumped amplifier was measured to be less than $\pm 2 \mathrm{~ns}$.

The preamplified $193 \mathrm{~nm}$ beam is subsequently sent through a $4 \mathrm{X}$ beam expander before it enters the electron beam pumped power amplifier (EPPA). The EPPA is a 50 $\mathrm{cm}$ long excimer laser head with a useful aperture diameter of $4 \mathrm{~cm}$ excited with $650 \mathrm{kV}, 85 \mathrm{kA}, 10 \mathrm{~ns}$ long electron beam pulses with a maximum repetition frequency of $1 \mathrm{~Hz}$. The design details of the EPPA are described in Ref. 19. The optimum gas fill of the EPPA was found to be a mixture consisting of $6.6 \mathrm{mbar} \mathrm{F}_{2}: 400 \mathrm{mbar}$ Ar: $4000 \mathrm{mbar} \mathrm{Ne}$. Although the short pulse energy directly after the preamplifier was $4 \mathrm{~mJ}$, the pulse energy after the EPPA (without pumping the active medium) was reduced to $1.5 \mathrm{~mJ}$ due to losses in optical elements and in air.

The EPPA increased the overall ultrashort pulse energy to $60 \mathrm{~mJ}$, with typically $5 \mathrm{~mJ}$ of time integrated ASE. This corresponds to an output energy density of $8 \mathrm{~mJ} / \mathrm{cm}^{2}$ which is very close to the $10 \mathrm{~mJ} / \mathrm{cm}^{2}$ maximum extractable energy density reported for a discharge pumped ArF amplifier in Ref. 20. This implies that the gain saturation characteristics of ArF are similar for discharge and electron beam pumping as it has been demonstrated for $\mathrm{KrF}$ in Ref. 6.

The pulse duration after the EPPA was measured with a multiple shot, Michelson-type autocorrelator based on two photon ionization of Xe. The current of the ionization chamber containing $10 \mathrm{mbar}$ of $\mathrm{Xe}$ as a function of the delay of the interferometer is presented in Fig. 2. The autocorrelation function shown here corresponds to a pulse duration of $710 \mathrm{fs}$ assuming a Gaussian pulse shape. We believe that this pulse duration is the result of pulse broadening presumably due to coherent pulse propagation 
through $\mathrm{O}_{2}$ in the air as it has been demonstrated in Ref. 12 , since the seen pulse duration is expected to be the same ( $300 \mathrm{fs}$ ) as that of the $707 \mathrm{~nm}$ dye laser, ${ }^{15}$ and the pulse broadening due to group velocity dispersion in the $\mathrm{CaF}_{2}$ windows of the excimer lasers is expected to be only $\sim 100$ fs. Consequently, it seems to be feasible, that the output power of the system could be increased to the $0.3 \mathrm{TW}$ level, by using beam tubes purged with $\mathrm{N}_{2}$ which would maintain the original seed pulse duration and result in a decrease in propagation losses.

In conclusion, we have developed an ultrahighbrightness ArF excimer laser system based on a spectrally compensated sum-frequency scheme as a seed pulse generator and an off-axis, double-pass, discharge pumped excimer preamplifier followed by an electron beam pumped power amplifier. The system generates pulse energies of up to $60 \mathrm{~mJ}$ with a pulse duration of $\sim 700 \mathrm{fs}$, and less than $10 \%$ ASE background.

The support of the Robert Welch Foundation Grant C-0586, and the Office of Naval Research is gratefully acknowledged. G. S. also acknowledges support from the OTKA Foundation of the Hungarian Academy of Sciences with Grant No. 3056, and a National Science Foundation Grant No. INT-9020541.

${ }^{1}$ B. Luther-Davies, E. G. Gamaliil, Y. Wang, A. V. Rode, and V. T. Tikhonchuk, Sov. J. Quantum Electron. 22, 289 (1992).

${ }^{2}$ J. H. Glownia, G. Arjavalingam, P. P. Sorokin, and J. E. Rothenberg, Opt. Lett. 11, 79 (1986).
${ }^{3}$ S. Watanabe, A. Endoh, M. Watanabe, and N. Sarukura, Opt. Lett. 13, 580 (1988).

${ }^{4}$ A. J. Taylor, C. R. Tallman, J. P. Roberts, C. S. Lester, P. H. Y. Lee, and G. A. Kyrala, Opt. Lett. 15, 39 (1990).

${ }^{5}$ S. Szatmari, F. P. Schäfer, E. Müller-Horsche, and W. Mückenheim, Opt. Commun. 63, 305 (1987).

${ }^{6}$ J. R. M. Barr, N. J. Everall, C. J. Hooker, I. N. Ross, M. J. Shaw, and W. T. Toner, Opt. Commun. 66, 127 (1988).

${ }^{7}$ T. S. Luk, A. McPherson, G. Gibson, K. Boyer, and C. K. Rhodes, Opt. Lett. 14, 1113 (1989).

${ }^{8}$ A. Endoh, M. Watanabe, N. Sarukura, and S. Watanabe, Opt. Lett. 14, 353 (1989).

${ }^{9}$ Th. Hofmann, T. E. Sharp, C. B. Dane, P. J. Wisoff, W. L. Wilson, F. K. Tittel, and G. Szabó, IEEE J. Quantum Electron. QE-28, 1366 (1992).

${ }^{10}$ H. Egger, T. S. Luk, K. Boyer, D. F. Muller, H. Pummer, T. Srinivasan, and C. K. Rhodes, Appl. Phys. Letl. 41, 1032 (1982).

${ }^{11}$ S. Szatmari and F. P. Schäfer, J. Opt. Soc. Am. B 6, 1877 (1989).

${ }_{12}^{12}$ J. H. Glownia, M. Kaschke, and P. Sorokin, Opt. Lett. 17, 337 (1992).

${ }^{13}$ I. V. Tomov, T. Anderson, and P. M. Rentzepis, Appl. Phys. Lett. 61, 1157 (1992).

${ }^{14}$ A. Tünnermann, C. Momma, K. Mossavi, C. Windolph, and B. Wellegehausen, IEEE J. Quantum Electron. (to be published).

${ }^{15} \mathrm{Th}$. Hofmann, K. Mossavi, F. K. Tittel, and G. Szabó, Opt. Lett. 17, 1691 (1992).

${ }^{16}$ T. E. Sharp, C. B. Dane, D. Barber, F. K. Tittel, P. J. Wisoff, and G. Szabó, IEEE J. Quantum Electron. 27, 1221 (1991); also F. K. Tittel, Th. Hofmann, T. E. Sharp, P. J. Wisoff, W. J. Wilson, and G. Szabo, Dye Lasers 25 Years, edited by M. Stuke (Springer, Berlin, 1992).

${ }^{17}$ G. Szabó, A. Müller, and Zs. Bor, Opt. Lett. 13, 746 (1988).

${ }^{18}$ S. Szatmari, G. Almasi, and P. Simon, Appl. Phys. B 53, 82 (1991).

${ }^{19}$ C. B. Dane, G. J. Hirst, S. Yamaguchi, Th. Hofmann, W. L. Wilson, R. Sauerbrey, F. K. Tittel, W. L. Nighan, and M. C. Fowler, IEEE J. Quantum Electron. 26, 1559 (1990).

${ }^{20} \mathrm{~K}$. Mossavi, Th. Hofmann, F. K. Tittel, and G. Szabó, Opt. Lett. 18, 435 (1993). 